\title{
Traumatic Chylothorax
}

National Cancer Institute

\section{Source}

National Cancer Institute. Traumatic Chylothorax. NCI Thesaurus. Code C101274.

Accumulation of lymph fluid in the pleural cavity as a result of thoracic trauma. 